\title{
POTASSIUM AND THE CARDIOGRAPHIC CHANGES IN DIABETIC ACIDOSIS
}

\author{
BY \\ C. B. HENDERSON \\ From the Cardiovascular Department, General Hospital, Newcastle-upon-7yne \\ Received June 6, 1952
}

This investigation was attempted in order to evaluate the cardiogram as a guide in estimating the level of potassium in the serum during, and when a patient is emerging from, diabetic acidosis. Shirley Smith and Hickling (1932) in a paper on cardiographic abnormalities in severe diabetes concluded that these changes represented mainly parenchymatous damage to the heart resulting from defective nutrition and that coronary disease, whether permanent or reversible, probably played a subsidiary role. Cardiographic changes in diabetic acidosis were described by Bellet and Dyer (1937) in a study of seventeen cases of coma and six of precoma; they listed lengthening of the Q-T interval, depression of the S-T segment, and inversion of the $T$ wave as the most frequent findings and alteration of the QRS complex as infrequent. Other changes since described are: slight alteration in the character and amplitude of the $P$ wave (Martin and Wertman, 1947); a prominent U wave (Ernstene and Proudfit, 1949); and cardiac arrhythmias (Bellet et al., 1950). Ernstene and Proudfit (1949) did not find any constant lengthening of the Q-T interval in the cases they studied and pointed out that the commonly present $U$ wave may cause apparent lengthening of the Q-T interval by partial fusion with the descending limb of the $T$ wave.

The cause or causes of the cardiographic changes have been the subject of controversy among many authors. Because of the rapid development and regression of the abnormalities, Bellet and Dyer (1937) suggested that they were the result of a transient electrolyte imbalance. In the series of Martin and Wertman (1947) no close correlation was found between the levels of serum potassium and the depression of the S-T segment and prolongation of the Q-T interval. They thought that very low T waves were associated with low levels of serum potassium. Tarail (1948) found that the changes in the cardiograms of three out of five patients did not correlate with the degree of hypopotassæmia. Bellet et al. (1950) concluded that "The Q-T interval was a crude guide to the degree of hypopotassæmia, although the $\mathrm{T}$ wave and $\mathrm{S}-\mathrm{T}$ changes were not reliable criteria of the degree of hypopotassæmia when comparisons were made in different patients; on the same patient, however, they correlated fairly well with the degree of hypopotassæmia."

Cardiographic changes similar to those found in dabetic acidosis, and associated with disturbances of potassium metabolism have provided further material for controversy concerning the role of serum potassium in their production. Steward et al. (1940), Stoll and Ninsnewitz (1941), and Perelson and Cosby (1949) described the cardiographic findings during the paralytic and recovery phases of familial periodic paralysis. Ernstene and Proudfit (1949) recorded the levels of potassium in the serum in a patient with Addison's disease who developed invertion of T waves, depression of S-T segments, and prominent $U$ waves after being treated with desoxycorticosterone acetate to excess. Perkins et al. (1950) reported cardiographic findings in one case and McAllen (1951) in three of chronic diarrhøea with hypopotassæmia. The former described lengthening of the Q-T interval while the latter thought there was little if any Q-T change. Both of these authors concluded that there was correlation between the $\mathrm{T}$ wave and the $\mathrm{S}-\mathrm{T}$ changes and the degree of hypo- 
potassæmia, but McAllen could not find any correlation between the level of potassium in the serum and the slightly prolonged Q-T interval. He considered that in most previous publications the Q-T interval had been overestimated by including the $\mathrm{U}$ wave in the measurement.

Examination of the evidence shows that of the potassium deficiency diseases the most profound cardiographic abnormalities are found in diabetic acidosis. Low levels of potassium in the serum in diabetic acidosis were associated with more severe abnormalities of cardiographic form than the same potassium levels in idiopathic steatorrhœa, for example. The most significant difference in the alteration of potassium metabolism in these two diseases is the rapidity of the change, and this will be discussed later.

\section{MATERIAL AND INVESTIGATION}

The present study is of ten consecutive cases of diabetic acidosis (four in coma and six in early coma) admitted into Newcastle General Hospital between September, 1950, and March, 1951. Eight patients were women, two were men, and their ages ranged between 12 and 59 years, with an average of 38 years. Two women died. Each case on admission had a blood sugar level above $275 \mathrm{mg}$. per $100 \mathrm{ml}$. and a carbon dioxide combining power below 22 volumes per cent.

Cardiogram, blood pressure, and venous blood for chemical analysis were taken in rapid sequence at intervals during and following the period of acidosis in each case. The carbon dioxide combining power, blood sugar and serum potassium levels were determined; the serum was separated within one hour of collection for the potassium analysis and always analysed by the same technician. The Jacobs and Hoffman (1931) method (normal range 16-20 mg. per $100 \mathrm{ml}$. was used for the potassium analysis and the accuracy of the method found to be $\pm 0.5 \mathrm{mg}$., using duplicate specimens.

Fifty-two cardiograms were taken on the Sanborn direct-writing cardiograph, with the patients supine in bed. The first cardiogram and blood specimen were taken within two hours of admission, but as several patients had been given large doses of soluble insulin subcutaneously before admission, records before treatment were not obtained in all. No patient was given digitalis or quinidine during the illness.

Examinations were performed post mortem on the two patients who died. Cardiac examination, before discharge from hospital in each of the patients who survived, showed no abnormality.

\section{Presentation of Cases}

The blood pressure, blood sugar and serum potassium levels are shown with each cardiogram in the illustrations, together with the times and dates.

Case 1 (Fig. 1). Mrs. G. B., aged 29 years, was admitted in early coma. On September 28, 1950, the serum potassium level was $10 \mathrm{mg}$. per $100 \mathrm{ml}$. and the cardiogram showed depression of S-T segments, inversion of $T$ waves and prominent $U$ waves. By October 8 , the serum potassium was $17.5 \mathrm{mg}$. and the cardiogram almost normal. There was no correlation between the cardiographic and serum potassium changes in two of the six cardiograms taken.

Case 2 (Fig. 2). Mrs. M. H., aged 35 years, was admitted in early coma. On October 6, 1950, the serum potassium was $16 \mathrm{mg}$. and the cardiogram showed tall and pointed $P$ waves and inverted T waves in leads II and III and in CR6. T waves in leads CR2 to 4 were tall and narrow. One and a half hours later the serum potassium was $9.5 \mathrm{mg}$. and except for inverted $\mathrm{T}$ waves in leads II and III the cardiogram was normal. On October 7, the serum potassium was $14 \mathrm{mg}$. and the cardiogram showed paroxysmal nodal rhythm, inverted $T$ waves and prominent $U$ waves. On October 8 , the serum potassium was unchanged and the cardiogram normal. The serum potassium and cardiographic changes did not correlate in this series.

Case 3 (Fig. 3). Mrs. I. D., aged 36 years, was admitted in early coma. On October 29, 1950 the serum potassium was $24.4 \mathrm{mg}$. and the cardiogram showed tall and pointed $P$ waves, depressed S-T segments, tall and peaked $T$ waves in leads CR1 and 4, and flat or inverted T waves in leads II, III, and CR6. Two hours later the serum potassium was $12 \mathrm{mg}$. and the cardiogram unchanged. On October 30, the serum potassium was $17 \mathrm{mg}$. and the cardiogram showed isoelectric $S-T$ segments and flat $T$ waves On October 31 , the serum potassium was unchanged and the cardiogram had reverted to normal. There was no correlation between the serum potassium and the cardiographic changes in this series. 


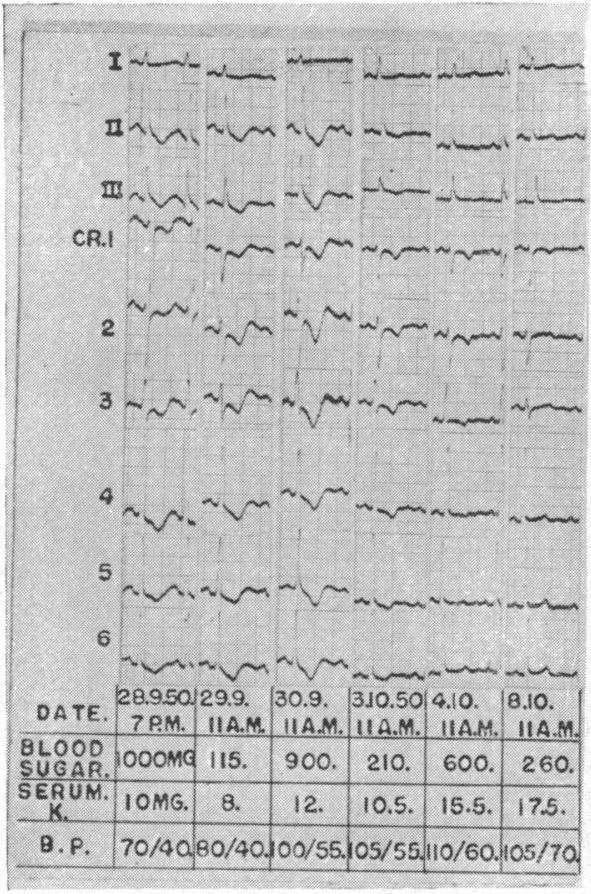

FIG. 1.

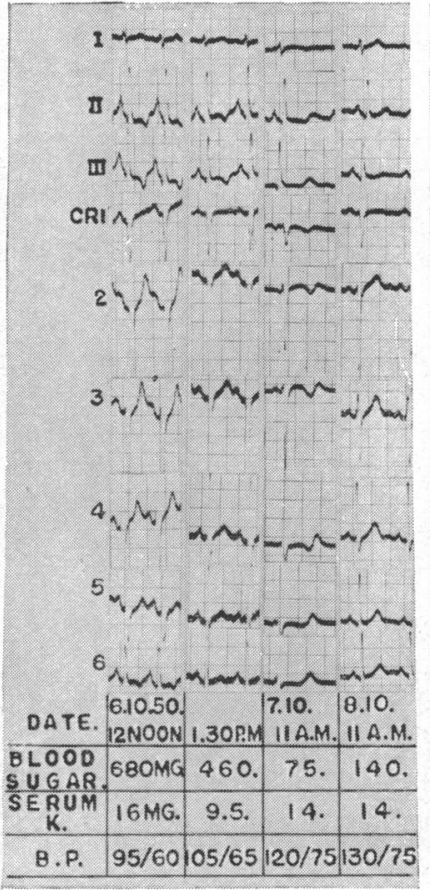

FIG. 2.

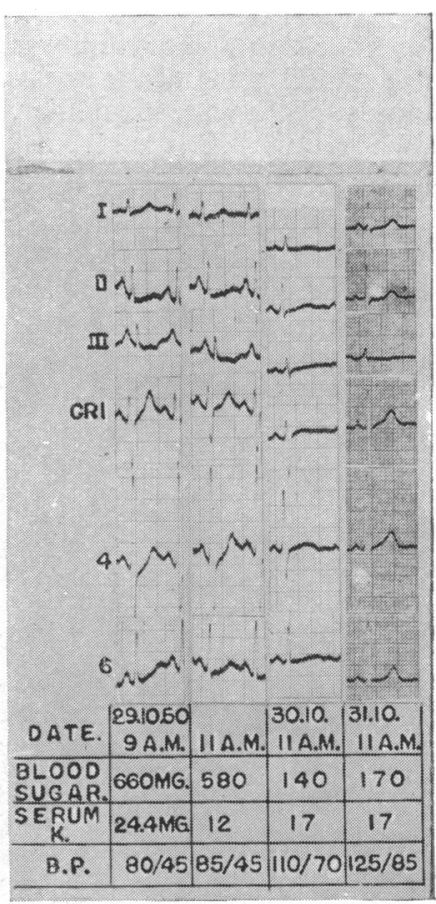

FIG. 3.

FIG. 1.-Diabetic acidosis. Case 1. S-T segments are depressed, T waves inverted and U waves prominent. Normal in 8 days.

FIG. 2.-Diabetic acidosis. Case 2. First tracing-tall and pointed $\mathbf{P}$ waves, inverted $\mathbf{T}$ waves in leads II, III, and CR6, and tall and narrow $T$ waves in CR2 to 4 . Third tracing-paroxysms of nodal rhythm, inverted $T$ waves and prominent $U$ waves. Normal in 3 days.

FIG. 3.-Diabetic acidosis. Case 3. Cardiographic abnormalities are similar to but not so severe as those seen in Fig. 2. Normal in 3 days.

Case 4. Miss M. M., aged 27 years, was admitted in coma. On November 2, 1950, the serum potassium was $15 \mathrm{mg}$. and the cardiogram was normal except for prominent $\mathbf{P}$ waves. Three hours later the serum potassium was unchanged and the cardiogram showed $\mathbf{S}-\mathbf{T}$ segment depression and small $\mathbf{T}$ waves. The next day the serum potassium had fallen to $13.5 \mathrm{mg}$. but the cardiogram had reverted to normal. On the following day the serum potassium also was normal. The cardiographic and serum potassium changes did not correlate in three of the five cardiograms taken.

Case 5 (Fig. 4). Mrs. F. F., aged 58 years, was admitted in coma. The serum potassium was $18 \mathrm{mg}$. and the cardiogram showed prominent $P$ waves, depressed $S-T$ segments, tall $T$ waves in $C R 1$ and inverted $T$ waves in the limb leads and in CR4 and 6. One hour later the serum potassium had fallen to $10 \mathrm{mg}$. and the cardiogram was unchanged. Two days later the serum potassium and cardiogram were normal. The cardiographic and serum potassium changes did not correlate in three of the four cardiograms taken.

Case 6. J. S., aged 12 years, was admitted in early coma. The serum potassium was $21 \cdot 5 \mathrm{mg}$. and the cardiogram showed depression of $\mathrm{S}-\mathrm{T}$ segments, flat or inverted $\mathrm{T}$ waves, and prominent $U$ waves. Three hours later the serum potassium had fallen to $12.5 \mathrm{mg}$. and the cardiogram was more abnormal. Two days later the serum potassium level and the cardiogram had returned to normal. The cardiographic and serum potassium changes did not correlate in two of the six cardiograms taken.

Case 7 (Fig. 5). Mrs. M. S., aged 34 years, was admitted in early coma. At noon on November 27, 1950 , the serum potassium and the cardiogram were normal. Four hours later the serum potassium had fallen to $12.5 \mathrm{mg}$. and the $\mathrm{T}$ waves of the cardiogram were smaller. On November 28 , the serum potassium had increased to $15.5 \mathrm{mg}$. but the amplitude of the $\mathrm{T}$ waves had further diminished and $U$ waves were evident. On November 29, the serum potassium and cardiogram were normal. The cardiographic and serum potassium changes did not correlate in one of the four cardiograms taken. 
Case 8 (Fig. 6). Mr. J. D., aged 39 years, was admitted in early coma. At 8 a.m. on December 26, 1950, the serum potassium was $13 \mathrm{mg}$. and the cardiogram showed auricular fibrillation with a ventricular rate of 150 a minute, depressed $S-T$ segments and inverted $T$ waves. Chest leads could not be taken because of the patient's tremor. Sinus rhythm returned the same day with the serum potassium at $15.5 \mathrm{mg}$. On December 30, the serum potassium level and the cardiogram were normal. The cardiographic and serum potassium changes did not correlate in one of the six cardiograms taken.

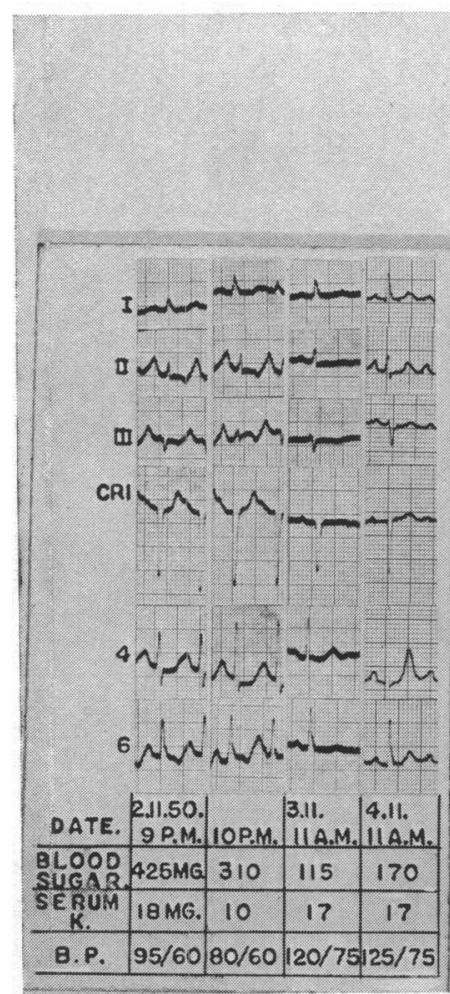

FIG. 4.

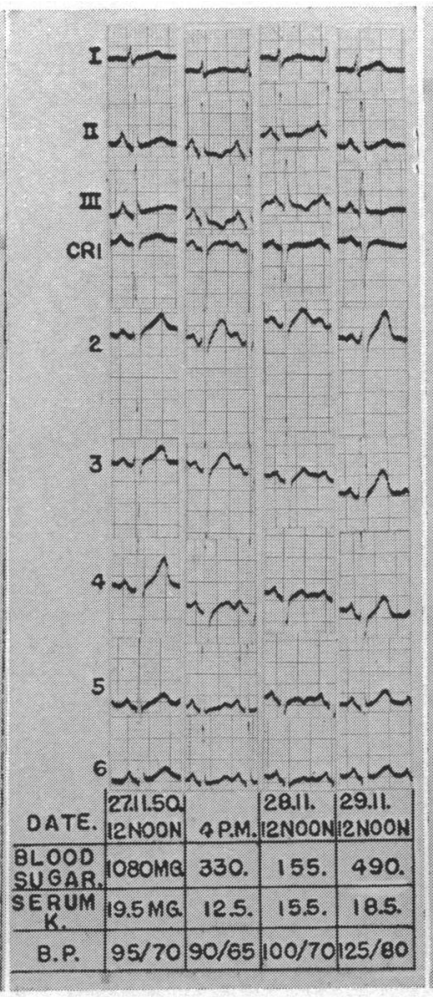

FIG. 5.

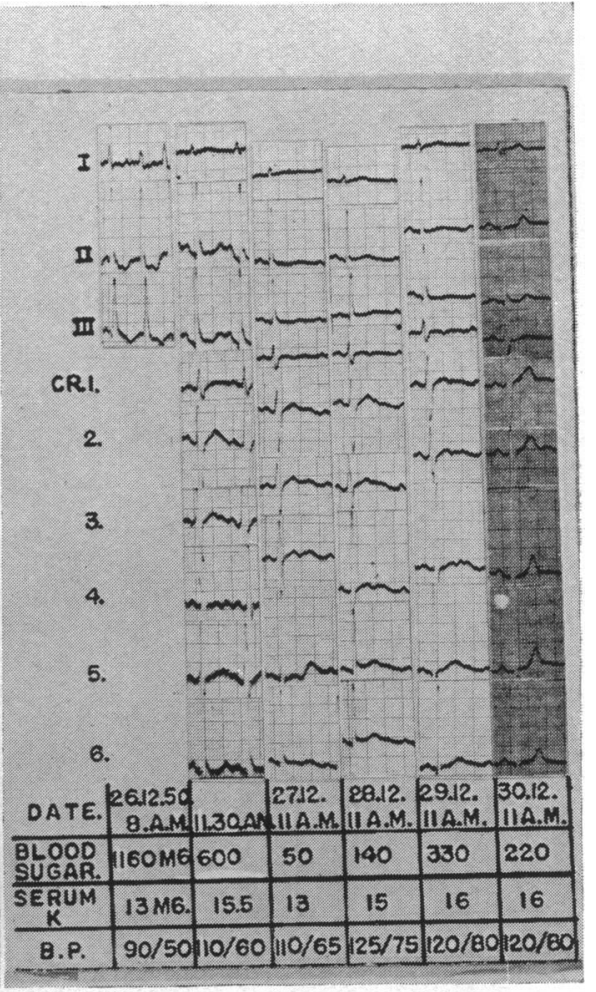

FIG. 6.

Fig. 4.-Diabetic acidosis. Case 5. Cardiographic abnormalities are similar to those seen in Fig. 3. Normal in 3 days.

Fig. 5.-Diabetic acidosis. Case 7. T waves are low or inverted in leads II, III, and the left chest leads. Normal in 3 days.

Fig. 6.-Diabetic acidosis. Case 8. First tracing-auricular fibrillation, S-T segment depression and inverted T waves. Second tracing-sinus rhythm. Normal in 5 days.

Case 9 (Fig. 7). Mrs. E. M., aged 50 years, was admitted in coma. At noon on January 23, 1951, the serum potassium was normal and the cardiogram showed auricular fibrillation with a ventricular rate of 130 a minute, wide QRS complexes, depressed S-T segments, and inverted T waves in the left chest leads. At 4 p.m. the cardiogram was unchanged but the serum potassium level had fallen to $13 \mathrm{mg}$. At 8 p.m. the serum potassium had further fallen to $10 \mathrm{mg}$. and the cardiogram showed considerable improvement and restoration of sinus rhythm. The cardiographic and serum potassium changes did not correlate in any instance.

The patient died two hours after the last cardiogram shown. At post-mortem examination the heart weighed $375 \mathrm{~g}$. and there was no macroscopic evidence of heart disease. The coronary arteries were normal. Histological examination showed loss of muscle striation but no muscle necrosis or increased fibrosis. 


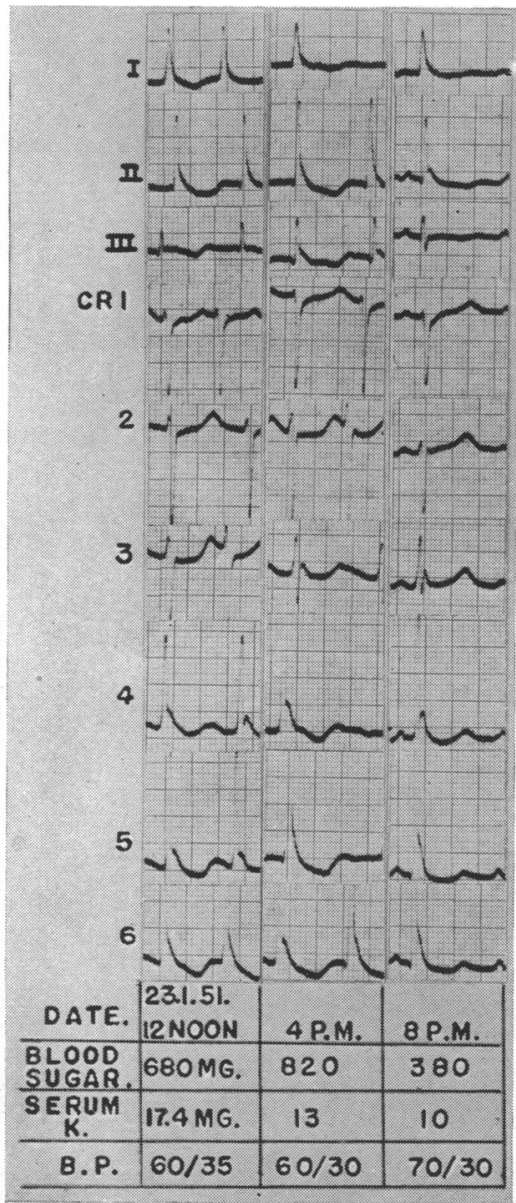

Fig. 7.-Diabetic acidosis. Case 9. First and second tracings-auricular fibrillation, S-T segment depression and flat or inverted $\mathbf{T}$ waves. Third tracing-sinus rhythm and larger $\mathrm{T}$ waves. Cardiogram much improved in 8 hours.

Case 10 (Fig. 8). Mrs. E. A., aged 59 years, was admitted in coma. At 7 p.m. on March 6, 1951, the serum potassium was $12 \mathrm{mg}$. and the cardiogram showed auricular fibrillation with a ventricular rate of 130 a minute, widening of QRS complexes, depression of S-T segments, and inversion of T waves. Three hours before death on March 10,1951, the serum potassium was $11.5 \mathrm{mg}$. and the cardiogram normal. The cardiographic and serum potassium changes did not correlate in five of the ten cardiograms taken. At post-mortem examination the heart weighed $420 \mathrm{~g}$. and the myocardium of the left ventrical showed multiple small areas of scarring. The coronary arteries were atheromatous but nowhere completely occluded. Histological examination showed diffuse fibrosis throughout the myocardium of the left ventricle. There was moist gangrene of the right foot and lower leg.

\section{DisCussion}

The present series of 10 cases again demonstrates the profound alterations that may occur in the cardiogram in patients during diabetic acidosis and when emerging from it. The cardiograms show that certain abnormalities occurred more frequently than in previous reports, e.g. cardiac arrhythmias, altered $\mathrm{P}$ waves, and prolonged QRS complexes; and a cardiographic pattern in uncomplicated diabetic acidosis has been brought to light. This pattern is seen in the first cardiograms in Figures 2, 3, and 4, and consists of tall and peaked $T$ waves in the right chest leads and flat or inverted T waves in leads II and III and in the left chest leads; thus showing simultaneously 


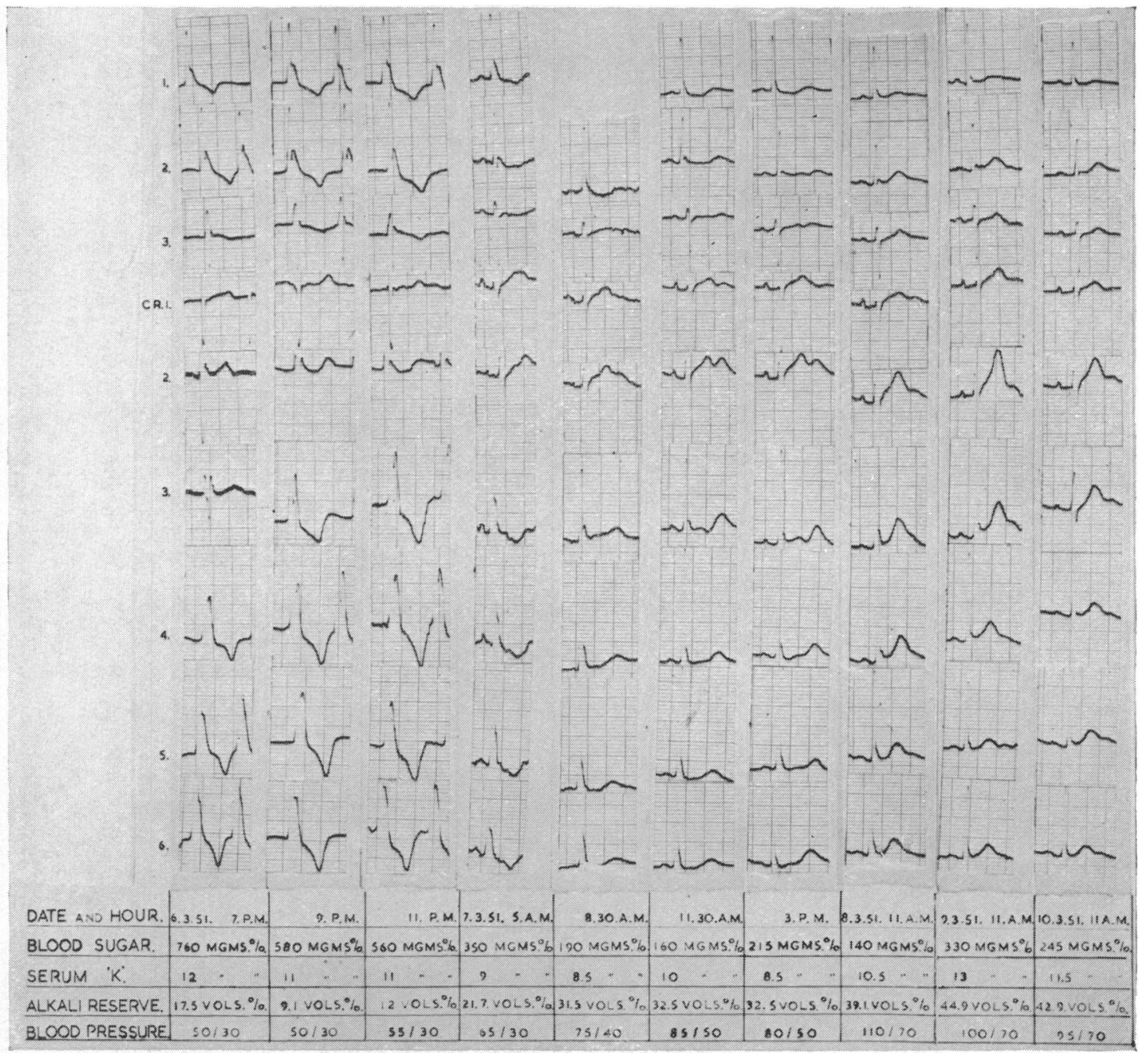

Fig. 8. -Diabetic acidosis. Case 10. First, second, and third tracings-auricular fibrillation, S-T segment depression and inverted $T$ waves. Fourth tracing - sinus rhythm. Fifth to seventh tracings-prominent $U$ waves. Normal in 5 days.

a combination of $\mathrm{T}$ wave abnormalities previously ascribed to a high concentration of potassium in the serum (tall $\mathrm{T}$ waves) and to a low concentration (flat and inverted $\mathrm{T}$ waves).

On the 42 occasions when abnormal features were present in the cardiograms, the degree of the abnormalities correlated with the levels of potassium in the serum in 21 instances and in 21 did not.

In general during the period when cardiographic abnormalities were increasing, the $P$ wave, QRS complex, S-T segment, and T wave abnormalities occurred together, and the increase in size of the $U$ wave occurred later. During recovery the $P$ wave, QRS complex, and S-T segment reverted to normal first, then the $T$ wave and finally the $U$ wave. This period of recovery varied from 24 to 184 hours with an average of 68 hours in the patients who recovered. Of the two patients who died, one (Case 10) showed almost complete return to cardiographic normality in 88 hours, and the other (Case 9) considerable improvement in 8 hours. In general, the more pronounced the abnormality, the slower was the return to normal. 


\section{The Possible Influence of Intracellular Potassium on the Cardiogram}

The oral or intravenous administration of potassium to a patient with diabetic acidosis will, after a variable time, produce improvement in an abnormal cardiogram. It would therefore appear that depletion of body potassium is of major importance in the production of the abnormalities. However, the data presented in this paper shows that in the 42 abnormal cardiograms taken, the degree of the abnormality correlated in only 21 instances with the level of potassium in the serum.

In the chronic diseases associated with a negative potassium balance such as nephritis and diarrhœa, in which a gradual fall in serum potassium concentration often occurs, the $\mathrm{S}-\mathrm{T}$ segment and $\mathrm{T}$ wave changes appear to correlate roughly with the serum potassium levels (Perkins et al., 1950 , and McAllen, 1951). As the loss of body potassium in these diseases is slow it is probable that the extracellular potassium is in equilibrium with, and reflects the intracellular concentration. It is now recognized, however, that during the rapid phases of abnormal potassium metabolism such as occur in diabetic acidosis, the serum potassium does not always reflect the intracellular concentration. Danowski et al. (1947) showed that before treatment of diabetic acidosis the intracellular potassium concentration may be low, while the serum potassium is at normal levels, or even above. With recovery from the acidotic state, as the potassium in the cells increases, so the potassium in the serum falls sharply.

Darrow and Miller (1942) produced depletion of body potassium and cardiac lesions (necrosis of muscle fibres and replacement by fibroblasts) in rats by diets low in potassium, and by repeated injections of desoxycorticosterone acetate. They found that resting rats, on a low potassium diet, showed little change in the concentration of serum potassium despite the fact that muscle potassium was considerably reduced and the diminution of muscle potassium was an index of the incidence of cardiac lesions.

Perkins et al. (1950) reported a patient who showed potassium deficiency resulting from chronic diarrhœa who had an abnormal cardiogram. Oral and intravenous potassium chloride produced some improvement in the cardiogram which was, however, still abnormal shortly before death. The myocardium showed histological changes similar to those described in rats depleted of potassium. The authors concluded that the lesions in the heart were due to potassium deficiency analogous to those seen in experiments on animals. The relationship between the low intracellular potassium and the myocardial necrosis in the rats, and the cardiographic changes and myocardial necrosis in the case described by Perkins et al. (1950) may reasonably be linked together in implicating the intracellular potassium as the important agent in the production of the cardiographic changes. Additional circumstantial evidence for this is provided in the case of familial periodic paralysis recorded by Stewart et al. (1940). At the height of muscle paralysis the serum potassium was $11 \mathrm{mg}$. and the cardiogram was grossly abnormal. Two and a half hours later, after giving potassium by mouth, the function of the muscle improved, and the cardiogram improved, but the level of potassium in the serum was unchanged. This would suggest that the potassium content of the skeletal and cardiac muscle was rising while the concentration in the serum remained unaltered, and that the cardiographic improvement resulted from an increase in the intracellular concentration and was not influenced by the serum concentration.

\section{SUMMARY}

Cardiographic abnormalities in ten cases of diabetic acidosis are described.

Cardiac arrhythmias occurred in four patients, three of whom showed transient auricular fibrillation and one showed transient nodal rhythm.

Tall and peaked $P$ waves were present in four patients and were best seen in leads II and III. Subsequent tracings in each patient showed a rapid transition to normal $\mathbf{P}$ waves during recovery.

Transient widening and splintering of the QRS complexes occurred in leads I and II and the left chest leads in two patients, both of whom died. 
Depression of the S-T segments were present at some time during the state of acidosis in eight patients.

Flat or inverted $\mathbf{T}$ waves in multiple leads were present in eight patients, six of whom also had depression of S-T segments.

$\mathrm{U}$ waves were abnormally large in six patients and small but easily identified in an additional three.

The Q-T interval could not be accurately measured in nine cases. In one (Case 7) in which accurate determination was possible, the corrected Q-T interval was normal.

A cardiographic pattern, not previously described, in diabetic acidosis, was seen in three cases and consisted of high peaked $T$ waves in the right chest leads and flat or inverted $T$ waves in the left chest leads.

Cardiographic recovery occurred within 24 to 184 hours in nine cases. Partial recovery occurred within 8 hours in one patient who died in coma.

There was no correlation between the level of the potassium in the serum and the degree of cardiographic abnormality in 21 of the 42 abnormal cardiograms taken.

The possible role of the intracellular potassium in the production of the abnormalities is discussed.

I wish to express my gratitude to Dr. George Richardson, physician in charge of the diabetic clinic, for his advice and for the ready access he gave me to patients under his care, and also to the staff of the Department of Pathology for the many biochemical estimations.

To Dr. W. G. A. Swan, I am particularly indebted for his encouragement and constructive criticism.

\section{REFERENCES}

Bellet, S., Dyer, W. W. (1937). Amer. Heart J., 13, 72.

, Steiger, W. A., Nadler, C. S., Gazes, P. C. (1950). Amer. J. med. Sci., 219, 542.

Danowski, T. S., Hald, P. M., Peters, J. P. (1947). Amer. J. Physiol., 149, 667.

Darrow, D. C., Miller, H. C. (1942). J. clin. Invest., 21, 601.

Ernstene, A. C., Proudfit, W. L. (1949). Amer. Heart J., 38, 260.

Jacobs, H. R. D., Hoffman, W. S. (1931). J. biol. Chem., 93, 685.

Martin, H. E., Wertman, M. (1947). Amer. Heart J., 34, 646.

McAllen, P. M. (1951). Brit. Heart J., 13, 159.

Perelson, H. N., Cosby, R. S. (1949). Amer. Heart J., 37, 1126.

Perkins, J. G., Petersen, A. B., Riley, J. A. (1950). Amer. J. Med., 8, 115.

Smith, K. S., and Hickling, R. A. (1932). Lancet, 1, 501.

Stewart, H. J., Smith J. J. (1940). Amer. J. med. Sci., 199, 789.

Stoll, B., Nisnewitz, S. (1941). Arch. intern. Med., 67, 755.

Tarail, R. (1948). Amer. J. Med., 5, 828. 